\title{
ELECTRON TRANSFER RATES OF ALKYL-FERROCENE MOLECULES FORMING INCOMPLETE MONOLAYER ON SILICON ELECTRODES
}

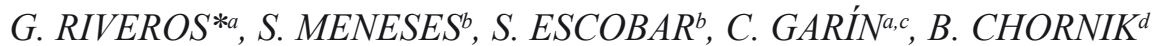 \\ ${ }^{a}$ Departamento de Química y Bioquímica, Facultad de Ciencias, Universidad de Valparaíso, Avenida Gran Bretaña 1111, Playa Ancha, Valparaíso, Chile. \\ ${ }^{b}$ Instituto de Química, Facultad de Ciencias Básicas y Matemáticas, Pontificia Universidad Católica de Valparaíso, Avenida Brasil 2950, Valparaíso, Chile. \\ 'Departamento de Química, Universidad Técnica Federico Santa María, Avenida España 1680, Casilla 110-V, Valparaíso, Chile. \\ ${ }^{d}$ Departamento de Física, Facultad de Ciencias Físicas y Matemáticas, Universidad de Chile, Casilla 487-3, Santiago 8370415, Chile.
}

(Received: June 9, 2009 - Accepted: December 7, 2009)

\begin{abstract}
This study shows the results obtained when binding alkyl ferrocene molecules on silicon surface forming incomplete monolayers. The electrodic surface functionalization is carried out by a two step procedure: First, a hydrosilytation reaction between a $\mathrm{Si}-\mathrm{H}$ surface and an alkenyl bromide active by white light; and then, the reaction of this surface with a monolithio ferrocene solution. Alkenyl bromide with different numbers of carbon atoms ( 3,5 and 10 carbon atoms) were employed in order to obtain propyl, pentyl and decyl ferrocene chains on the electrodic surface. The samples of modified silicon were analyzed by X-ray photoelectron spectroscopy (XPS) and electrochemical measurements confirming the presence of ferrocene molecules on the electrode surface. The results obtained show that in each and every case, there were incomplete monolayers on the silicon surface, ranging from $16 \%$ to $42 \%$, depending on the alkenyl bromide employed in the synthesis. AC voltammetry was employed to determine the kinetic of the electron transfer between ferrocene molecules and the silicon electrode. However, the rate constant is not influenced by the length of the alkyl chain, and is usually constant $\left(2.01 \mathrm{~s}^{-1}-3.66 \mathrm{~s}^{-1}\right)$. The result above is due to the electron transfer process which is determine by electron hopping in a regime of bonded diffusion and not by a long-rate electron transfer such as in a full compact monolayer.
\end{abstract}

Keywords: Silicon electrode; redox monolayers; AC voltammetry; electron transfer.

\section{INTRODUCTION}

The formation of functionalized organic monolayers on silicon surface has been the object of numerous studies because these interfaces can be used in different procedures such as: semiconductor-molecule electronic devices, photovoltaic application and biological sensing ${ }^{1,2}$. One of the main pathways for the formation of these monolayers is through of a covalent bond between the silicon and a carbon atom. This $\mathrm{Si}-\mathrm{C}$ bond has usually been produced by ultra high vacuum (UHV) techniques ${ }^{1,3,4}$ or by radicalary processes ${ }^{1,5}$. The latter, commonly named hydrosilylation, has been thoroughly studied due to its relatively low operation cost. The general mechanism of these reactions involves the double bond insertion into the $\mathrm{Si}-\mathrm{H}$ surface $^{1}$, where the double bond homolytic cleavage is carried out by reaction with peroxides ${ }^{6,7}$, by UV irradiation $^{8,9}$, by thermal activation ${ }^{10,11}$, by electrochemical processes ${ }^{12-16}$ or by white light irradiation ${ }^{17-20}$. High quality self-assambled monolayers (SAMs) have been obtained through these procedures. Usually, the binding of the functionalized redox molecules on silicon (i. e. porphyrins) includes successive steps of synthesis, separation, and characterization of the molecules, prior to their binding on the semiconductor surface ${ }^{21-23}$, hindering the study of these systems. An alternative to the above mentioned, is the employment of commercial molecules ${ }^{17-19,24-26}$, but the number of available systems is very limited. An alternative pathway is achieved by step by step modification stages on the silicon surface, similar to that employed by Fabre et al. ${ }^{27}$. In this case, following a reaction sequence on the silicon surface, the latter is modified with an alkyl chain which is latter functionalized with a ferrocene molecule. In the same manner, we have achieved a new route which will enable to modify the silicon surface with ferrocene molecules ${ }^{28}$. The procedure includes a hydrosilylation reaction (activated by white light) between an alkenyl halide and the hydride-terminated silicon surface, and the following reaction of this modified surface with monolithio-ferrocene (figure 1). This last step is similar to the one employed by Dietz et al. ${ }^{29}$ in the synthesis of alkyl ferrocenes. Using this procedure, alkyl chains functionalized with ferrocene molecules bonded to the silicon surface by means of a stable $\mathrm{Si}-\mathrm{C}$ bond have been obtained. The advantage of this procedure is that it employs different alkenyl halides during its modifying process, thus originating structures with different properties. Thus, this new, simple, and versatile synthesis method for the silicon modification with different ferrocene molecules can help to carry out new investigation in this field. However, the main problem of this procedure is that incomplete monolayers are obtained, showing that this method must be improved in some aspect, like quality of dry-box and source of light ${ }^{28}$.

On the other hand, investigations carried out in this field, generally show contradictory results, even in very similar systems. This can be because generally the process of percolation and bonded diffusion (and others superficial process, like interfacial solvation, ion-pairing, and interfacial potential distribution) are not considered when the reported results are analyzed.

This way, this study shows the results obtained in the silicon modification with alkyl chains functionalized with ferrocene molecules. Different length alkenyl halides were employed for the formation of various alkyl-ferrocene chains on silicon and therefore enabling us to study the dependence of the electron-transfer rates between the semiconductor and the ferrocene molecule with the chain length. Furthermore, we used the effect of formation of incomplete monolayers to study the effect of this parameter in the electrochemical response of these systems, showing important differences with those where compact monolayers are obtained. Thus, effects of bonded diffusion and electron hopping between adjacent molecules, that are not considered when compact monolayers are formed, were determinant in our systems.

\section{EXPERIMENTAL SECTION}

\subsection{Silicon cleaning}

p-Si(100) (International Wafer Service, $5-10 \Omega \mathrm{cm}$ ) was used in this study. Prior to the surface modification, the formation of reactive hydride-terminated silicon surface is necessary. Thus, the silicon wafers were cut with a diamond and were cleaned with a hot "piranha" solution $\left(\mathrm{H}_{2} \mathrm{SO}_{4}: \mathrm{H}_{2} \mathrm{O}_{2} 3: 1\right.$ at $\left.60-80^{\circ} \mathrm{C}\right)$ during a time span of 40 minutes. Following this, the samples were rinsed with deionizated water and treated with $2 \%$ aqueous HF. Finally, the samples were again washed with deionizated water, dried with an argon stream, and stored in a dry-box for their usage.

\subsection{Surface modification}

For the $\mathrm{Si}-\mathrm{C}$ bond formation and later functionalization with ferrocene, the following procedure was employed. First, was the reaction between the hydride-terminated silicon surface and the alkenyl halide (hydrosilylation reaction) activated by white light ${ }^{20}$. Following this, the modified surface was put in a monolithio-ferrocene solution, previously synthesized according to prior studies ${ }^{30-31}$. In order get different length alkyl-ferrocene chains, alkelyn halide of different number of carbon atoms were employed: 3-bromo 1propene; 5-bromo 1-pentene; and 10-bromo 1-decene. This way, surfaces modified with propyl (3 carbon atoms) pentyl (5 carbon atoms) and decylferrocene (10 carbon atoms) were obtained. This is summarized in figure 1.

Following this, the samples were successively washed with acetonitrile and dichloromethane in order to eliminate the rest of organic and organometallic compounds that remain on the electrode surface without reacting. 


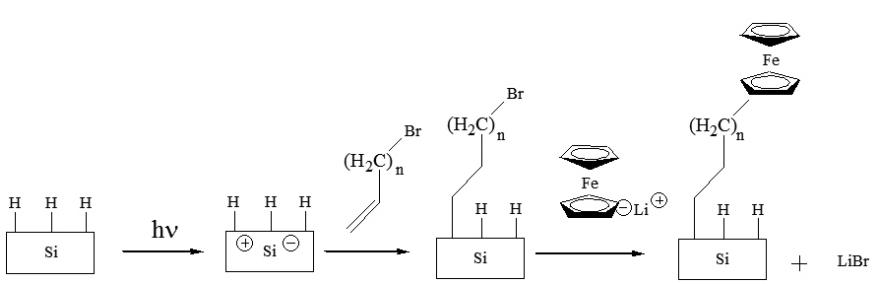

Figure 1: Sequence following for the silicon surface modification with redox molecules derived from ferrocene. First, the silicon surface is activated with white light and then react with the corresponding alkenyl bromide, where $\mathrm{n}=1$ for the 3-bromo 1 -propene, $\mathrm{n}=3$ for the 5 -bromo 1 -pentene, and $\mathrm{n}=8$ for the 10-bromo 1-decene. Finally, the modified surface reacts with monolithioferrocene forming the alkyl chain functionalized.

\subsection{Surface characterization}

The modified surface was characterized through $\mathrm{x}$-ray photoelectron spectroscopy (XPS). XPS measurements were carried out with a Perkin Elmer 1257 electron spectrometer which included an ultra high vacuum chamber and a hemispherical electron energy analyzer. The $\mathrm{X}$-ray source has two anodes: $\mathrm{Al}$ and $\mathrm{Mg}$, providing unfiltered $1486.6 \mathrm{eV}$ and $1253.6 \mathrm{eV}$ photons, respectively, which belong to the main $\mathrm{K} \alpha$ line. Binding energy calibration was achieved by using the peak of spontaneous carbon, fixing it at $284.8 \mathrm{eV}$.

\subsection{Electrochemical characterization}

All the electrochemical measurement were carried out with a $\mathrm{CH}$ Instrument potentiostat $(\mathrm{CHI} 604 \mathrm{C}$ ) in a conventional three electrodes electrochemical cell: working electrode (silicon and silicon modified electrodes); counter electrode (platinum wire); and reference electrode $\left(\mathrm{Ag} / \mathrm{Ag}^{+}\right)$. All the potentials shown in this study are related to this reference electrode. The measurements were carried out in a $0.1 \mathrm{M}$ tetraethylammonium perchlorate solution in anhydrous acetonitrile under dark in a Faraday's cage.

To determine the redox kinetic of the layers formed on the silicon electrodes, we employed the method developed by Creager et al. ${ }^{32}$. In this procedure, AC voltammetry measurements $(10 \mathrm{mV}$ amplitude $)$ are carried out at different frequency values. Usually, the $\mathrm{AC}$ voltammograms present a maximum value of AC current ( $\mathrm{I}$ ) which correspond to a faradaic process. The background current $\left(\mathrm{I}_{\mathrm{bg}}\right)$ represents the zone where non-faradaic processes take place. The $\mathrm{AC}$ voltammograms depend on the frequency employed, as a result of the redox kinetic of the studied systems. Plots $\mathrm{I}_{\text {peak }} / \mathrm{I}_{\mathrm{bg}} \mathrm{vs} \log$ (frequency) are obtained from these measurements. These curves are fitted with a suggested equivalent circuit $^{32}$ that contains faradaic and non-faradaic components. The resulting function includes as fitting parameters, the kinetic constant of the redox process $(\mathrm{k})$, the system capacity $(\mathrm{C})$ as well as the coverage of the surface $(\Gamma)$.

\section{RESULTS AND DISCUSSION}

3.1 XPS analysis

XPS measurements were carried out to confirm the presence of ferrocene molecules attached to the silicon surface through the different hydrocarbonated chains. Figure 2 shows a high resolution XPS spectrum of a silicon sample modified with propyl ferrocene (a) and pentyl ferrocene (b) in the Si 2p region. For curve fitting in this spectrum we used a model with three peaks (three different adjustable values for the chemical shift) and considered that each $\mathrm{Si}$ $2 p$ line is a doublet because of spin-orbit splitting. Therefore, the curve fitting calculation involves six peaks but the free parameters are only for three peaks, because all the $2 p_{1 / 2}$ levels are constrained to have $0.6 \mathrm{eV}$ higher binding energy than the corresponding $2 \mathrm{p}_{3,2}$ levels, and the peak areas of the $2 \mathrm{p}_{1 / 2}$ levels are exactly $1 / 2$ of the corresponding $2 p_{32}$ levels. Besides, both levels have the same line width and the same proportion between gaussian and lorentzian functions. The result of this fitting is: $2 \mathrm{p}_{3,2}$ levels located at $98.8 \mathrm{eV}$. $(\mathrm{Si}(0)), 99.5 \mathrm{eV}$ $(\mathrm{Si}(1+))$ and $102.1 \mathrm{eV}(\mathrm{Si}(4+))$. The $2 \mathrm{p}_{1 / 2}$ levels do not provide any additional information and are not used for identification.
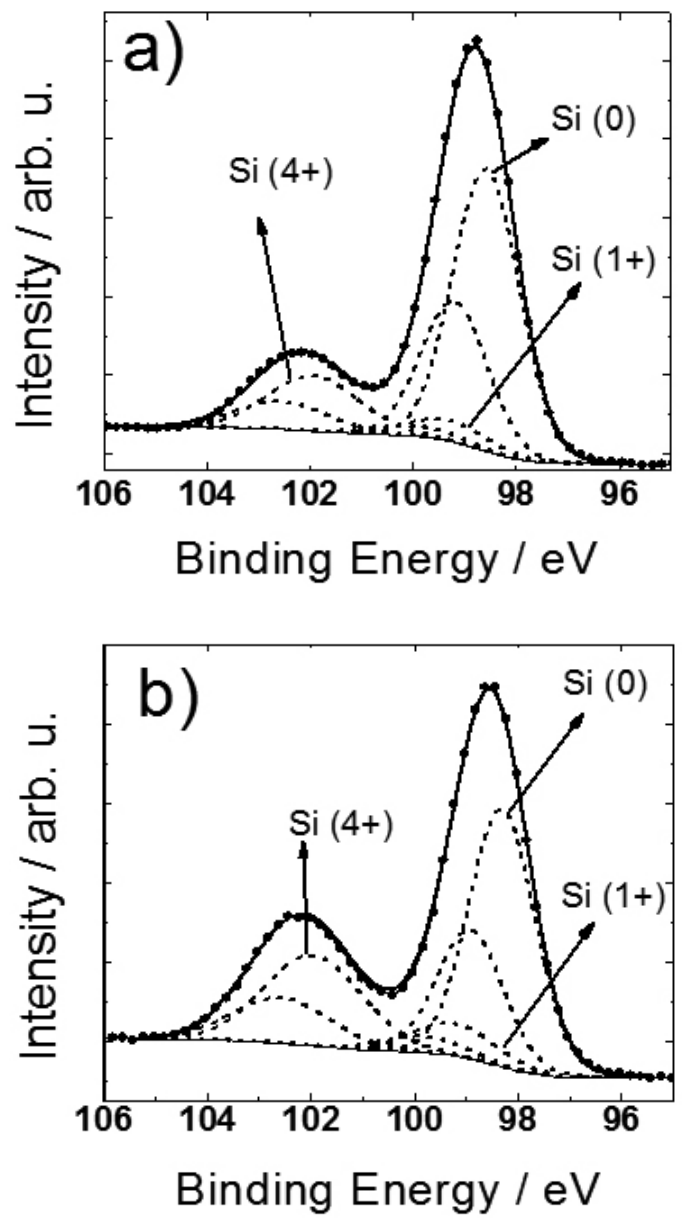

Figure 2: XPS spectrum in the Si $2 p$ region for a silicon sample modified with a) propyl-ferrocene and b) pentyl-ferrocene. Dotted lines are $2 p_{3,2}$ and $2 \mathrm{p}_{1 / 2}$ states. Continuous-circle line is the sum of all the above plus the background.

The peak at $102.1 \mathrm{eV}$ corresponds to oxidized silicon, in agreement with Dreiner et al..$^{33}$. We believe that in our experiment, it is produced during or after the modification process, as reported by Karadas et al. ${ }^{34}$. The presence of silicon oxide is not rare in this type of sample, as demonstrate the results obtained by other authors in similar conditions ${ }^{17}$, 18 . Furthermore, the XPS analyses are not realized immediately on fresh samples, allowing the formation of silicon oxide during the storage time. On other hand, the peak observed at $98.8 \mathrm{eV}$, corresponds to the $\mathrm{Si}-\mathrm{Si}$ bond present in the bulk of the sample, in agreement with Cardinaud et al..$^{35}$. The small peaks observed at $99.4 \mathrm{eV}$ can be assigned to $\mathrm{Si}(+1)^{33}$. These signals can be interpreted as a $\mathrm{Si}-\mathrm{C}$ bond or as a $\mathrm{Si}-\mathrm{H}$ bond, but the latter can be disregarded because of $\mathrm{SiO}_{2}$ formation (as indicated above).

Figure 3 shows the XPS spectrum for the Fe $2 p$ region for both samples. A curve fitting calculation gives three peaks, located at approximately $707.7 \mathrm{eV}$, $709.9 \mathrm{eV}$ and $714.8 \mathrm{eV}$ respectively. 

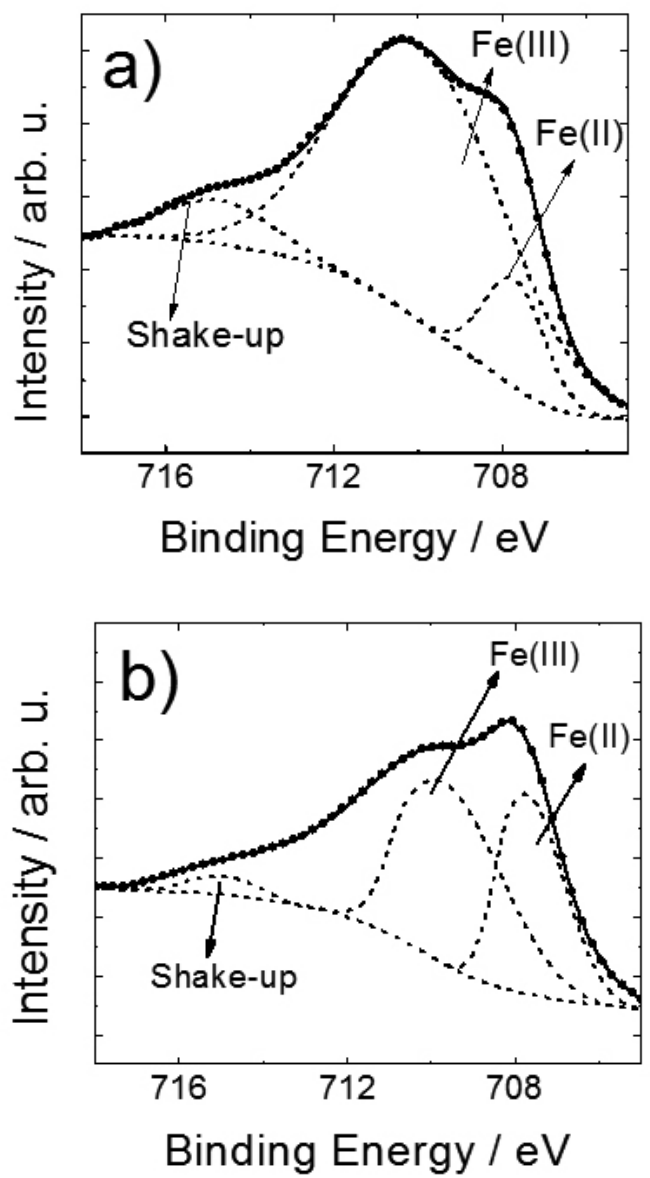

Figure 3: XPS spectrum in the Fe $2 p$ region for a silicon sample modified with a) propyl-ferrocene and b) pentyl-ferrocene. Dotted lines are experimental measurements. Continuous-circle line is the sum of all the above plus the background.

Following Fischer et al. measurements ${ }^{36}$, peak at 707.7 can be ascribed to ferrocene molecules ( $\mathrm{Fe}(\mathrm{II}))$. On other hand, peak at $709.9 \mathrm{eV}$ may correspond to $\mathrm{Fe}$ (III) species derived from ferrocene. The presence of Fe (III) species is not rare in this type of sample and could be the result of ferrocenium molecules resulting from the manipulation of the samples ${ }^{17-19}$ or from the exposition of ferrocene molecules to $\mathrm{X}$-rays ${ }^{37}$. Peak at $714.8 \mathrm{eV}$ is attributed to a shake-up satellite of $\mathrm{Fe}$ (III).

Thus, through XPS analysis, the presence of ferrocene molecules on the silicon surface is confirmed.

\subsection{Electrochemical analysis}

Figure 4 shows the Mott-Schottky plot obtained at a high frequency for hydride-terminated silicon electrodes in a $0.1 \mathrm{M}$ tetraethylammonium perchlorate solution in acetonitrile anhydrous. In this figure, a narrow lineal region can be observed between 0.0 and $-0.2 \mathrm{~V}$. However, it can be observed that lines obtained at different frequency values have a same intercept in the abscissa potential $(\sim 0 \mathrm{~V})$ and that they have a negative slope, whose values vary as does the frequency used in the measurement. The above is the typical behavior of a p-type semiconductor, confirming the nature of the employed electrode. From the slope value, the carrier density $\left(5.14 \times 10^{14} \mathrm{~cm}^{-3}\right)$ was calculated, coinciding with the value informed by the supplier. Using the same procedure, the flat band potential (abscissa potential interception, near to -0.03 $\mathrm{V}$ ) and the edge of the valence band (near to $0.09 \mathrm{~V}$ ) were obtained.

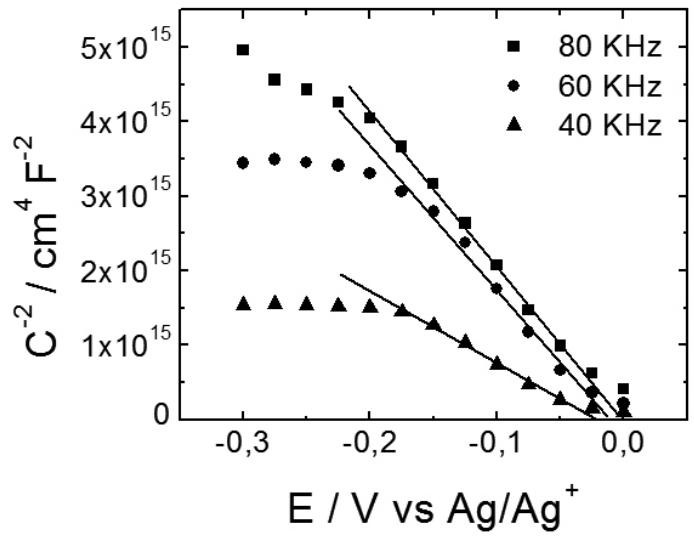

Figure 4: Mott-Schotky plots of the hydride-terminated silicon electrode at different frequencies in a $0.1 \mathrm{M}$ tetraethylammonium perchlorate solution in anhydrous acetonitrile.

Figure 5 shows the ciclyc voltammograms of the silicon samples modified with ferrocene molecules bonded to the electrode surface by alkyl chains of 3 , 5 , and 10 carbon atoms. In each case, a quasireversible electrochemical process can be observed. In every case, the redox potential (determined by the average value between the anodic peak potential and the cathodic peak potential) is situated inside the valence band of the silicon (table 1, figure 6).

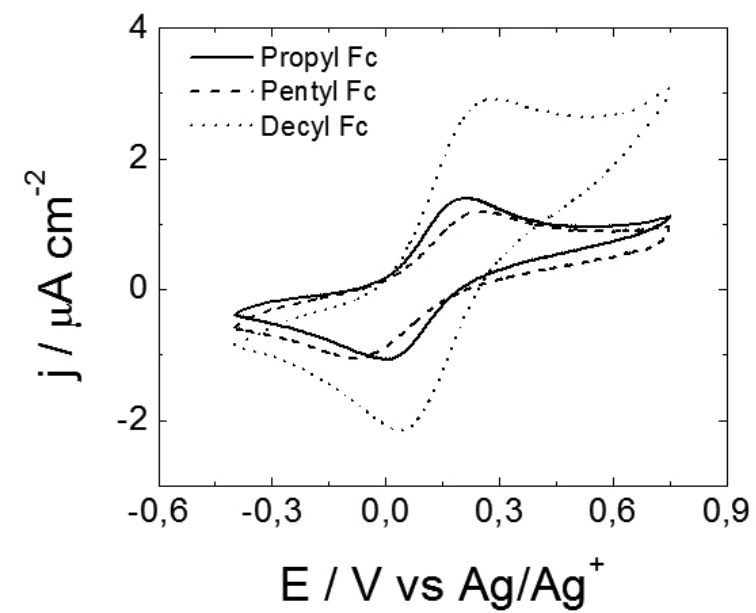

Figure 5: Cyclic voltammograms of the silicon surfaces modified with different alkyl chains functionalized with ferrocene molecules in $0.1 \mathrm{M}$ tertaethylammonium perchlorate solution in anhydrous acetonitrile. Scan rate $=100 \mathrm{mV} \mathrm{s}^{-1} . \mathrm{Fc}=$ ferrocene molecule.

The quasireversible response is a result of the proximity of the silicon valence band with the redox potential of the molecules bonded on the silicon surface. Considering that for a $\mathrm{p}$-type semiconductor in a desertion regime, the oxidation process can only be observed due to the fact that it is not energetically limited (hole injection from the silicon to the available energy levels of the reduced molecules). 


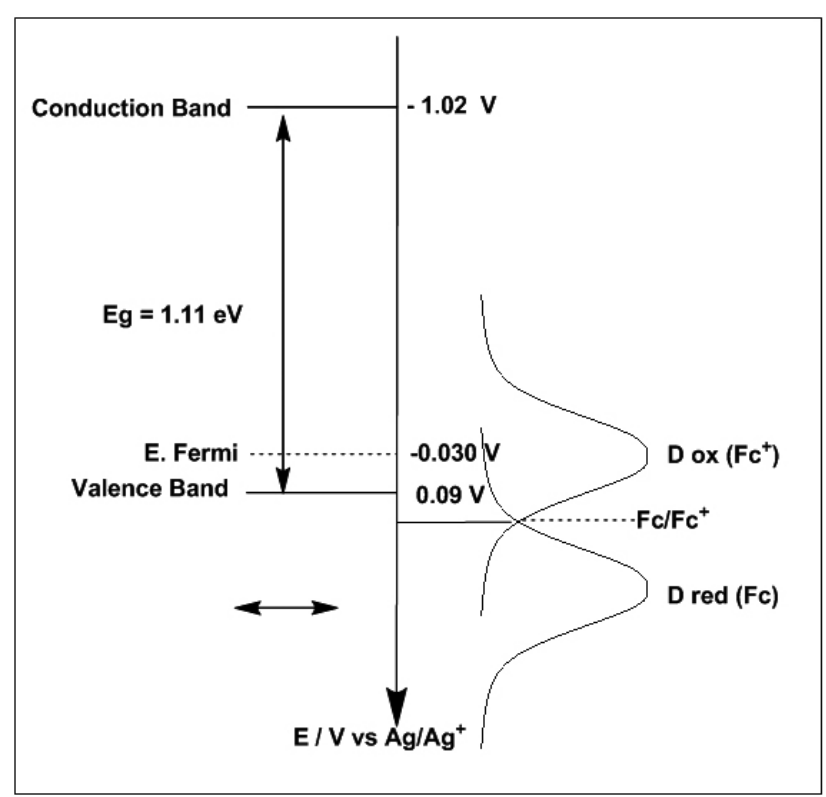

Figure 6: Energy diagram for p-type silicon (100) and the molecules derived of ferrocene.

On the other hand, the reduction process would only happen if the redox potential of the molecules was more positive than the edge of the substrate valence band (just as the studied systems) or if the semiconductor were to be in an inversion regime (electron injection from the silicon to the available energy levels of the oxidized molecules). Thus, in the case of the systems under study, the flux of electrons and holes between the oxidized and reduced states of the molecules and the silicon valence band is not energetically limited, but depends on the free state of the molecules.

The above mentioned, is reflected in the redox properties of the systems, which are summarized in Table 1 . However, in every studied case the relationship $\mathrm{I}_{\text {anodic peak }} / \mathrm{I}_{\text {cathodic peak }}$ was greater than 1 , which is coherent with the energetics of the studied systems

Table 1: Redox properties of the molecules bonded to the p-type silicon surface (100).

\begin{tabular}{|c|c|c|c|}
\hline $\mathrm{E}_{\text {redox }} / \mathrm{V}$ & $\mathrm{E}_{\text {redox }} / \mathrm{V}$ & $\Delta \mathrm{E}_{\mathrm{p}} / \mathrm{V}$ & $\Gamma / \%$ \\
\hline Propyl-Fc & 0.102 & 0.210 & $15.7-22.6$ \\
\hline Pentyl-Fc & 0.094 & 0.313 & $17.5-25.5$ \\
\hline Decyl-Fc & 0.155 & 0.227 & $37.6-41.7$ \\
\hline
\end{tabular}

In order to determine the surface coverage, the electrical charge integration of the oxidation process obtained from the previous voltammograms was employed. However, in the anodic scan, the oxidation of the substrate can also take place. Thus, this process was deconvolutionated using 3 gaussian peaks: one, corresponding to the ferrocene oxidation and the other two, to the substrate oxidation. In order to obtain the real coverage, it is necessary to consider the ferrrocene molecule diameter $(6.4 \AA)$ and assume that it is similar to the diameter of the molecules employed in the modification. Furthermore, it is necessary to consider the silicon atom's diameter $(2.64 \AA)$, its lattice parameter $(5.43 \AA)$ and the total number of silicon atoms present in an ideal flat surface with (100) orientation. Assuming the above mentioned, when one ferrocene molecule is placed on a silicon surface atom, because of the steric effect, there will be nine atoms of silicon covered. This way, the total number of ferrocene molecules to consider on a monolayer with an ideal flat surface is $7.53 \times 10^{13}$ molecules $\mathrm{cm}^{-2}$. By taking this quantity for one monolayer, and applying Faraday's law for the integration of the electrical charge of the molecules bonded to the silicon surface, it is possible to determine the real coverage on the modified electrodes. The results are shown in Table 1. As can be observed in every case, incomplete monolayers are formed

The relatively high coverage obtained in the case of the decyl-ferrocene molecule, is due to the synthesis method employed. In order to modify the silicon, three different alkenyl bromides were used: 3-bromo 1-propene; 5- bromo 1-pentene; and 10-bromo 1-decene. All these compounds are volatile, except for the 10-bromo 1-decene that has an elevated boiling point. Because the activation is carried out though white light, the heat of the lamp evaporates a great amount of these compounds, producing an inefficient modification. However, in the case of the 10-bromo 1-decene, the evaporation is not important; resulting in a more efficient surface modification.

\subsection{Electron transfer rates}

In order to determine the rate of electron-transfer in these systems the method developed by Creager et al. ${ }^{32}$ was employed. This method consists in carrying out $\mathrm{AC}$ voltammetry measurements at different frequencies and its subsequent analysis through an equivalent circuit so as to obtain the transference function. Numerous systems have employed this procedure in order to determine the electron-transfer kinetic in redox-active SAMs, including metallic $^{38-41}$ and semiconductor ${ }^{21,42}$ electrodes. For a reversible reaction, the position of the peak in the $\mathrm{AC}$ voltammogram provides information about the formal potential of the surface redox reaction, while the magnitude of the AC peak current provides information about the amount of redox-active material on the electrode. Furthermore, changes in the magnitude of the AC peak current with the changing frequency of the applied $\mathrm{AC}$ voltage provide information about the rate of electron transfer between the redox molecules and the electrode. Figure 7 shows the AC voltammograms at three different frequencies for the three systems included in this study.

In those measurements carried out a low frequency $(500 \mathrm{mHz})$, a maximum of AC current corresponding to the oxidation/reduction of ferrocene molecules on the silicon electrode, can be observed. As the frequency increases, the maximum of $\mathrm{AC}$ current diminishes until practically disappearing (frequency $>50 \mathrm{~Hz}$ ). From these results, plots $\mathrm{I}_{\mathrm{at}} / \mathrm{I}_{\mathrm{b}}$ vs $\log$ (frequency) can be obtained. $\mathrm{I}_{\text {peak }}$ is equivalent to the maximum $\mathrm{AC}$ current value obtained from the $\mathrm{AC}$ voltamograms, and $\mathrm{I}_{\mathrm{bg}}$ is equivalent to the current value where no electrochemical process can be observed.

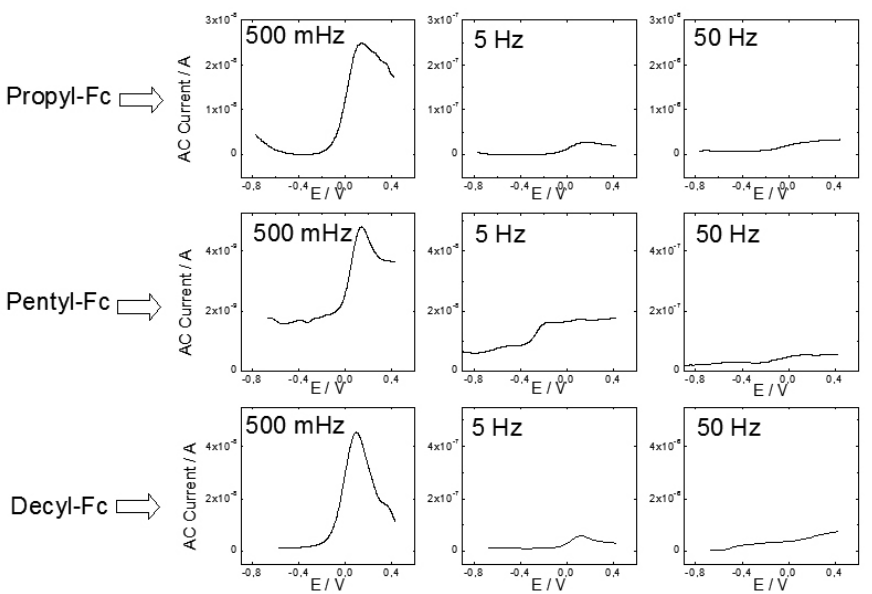

Figure 7: AC voltammograms at different frequencies for $\mathrm{p}-\mathrm{Si}(100)$ samples modified with propyl, pentyl and decyl ferrocene.

The potential value, in which $\mathrm{I}_{\text {peak }}$ is determined, is practically the same as the redox potential determined by cyclic voltammetry, thus confirming that these measurements are correspondent. By means of a fitting of the experimental results with a transfer function obtained from a suggested equivalent circuit ${ }^{32}$, the constant kinetic as well as the coverage and the capacity of the studied systems can be achieved. Figure 8 shows the experimental results and the fitting carried out on the latter in the systems developed in this study. 

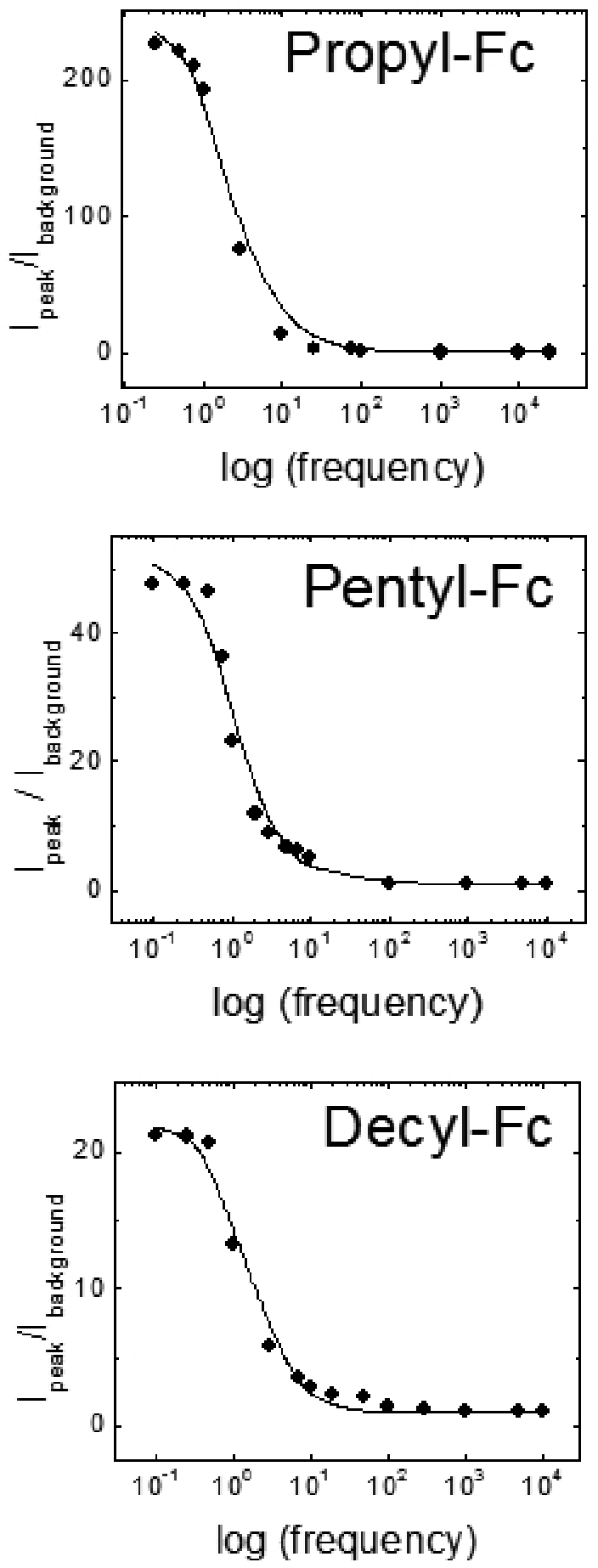

Figure 8: Plots $\mathrm{I}_{\text {peak }} / \mathrm{I}_{\text {background }}$ vs $\log$ (frequency) of silicon samples modified with propyl, pentyl y decyl ferrocene. The experimental results (circles) were fitted (continuous line) according to the equivalent circuit describe in the literature ${ }^{32}$.

Table 2 presents the values of each parameter employed in the fitting of the different silicon-molecule systems presented in this study.
Table 2: Results obtained for the kinetic constant, capacity and coverage for the different modified silicon samples according to the fitting realized in figure 8 .

\begin{tabular}{|c|c|c|c|}
\hline Molecule & $\begin{array}{c}\text { Kinetic } \\
\text { constant } \\
/ \mathbf{s}^{-1}\end{array}$ & $\begin{array}{c}\text { Capacity x } 10^{9} \\
/ / F \text { cm}^{-2}\end{array}$ & $\Gamma / \%$ \\
\hline Propyl-Fc & 3.66 & 1.10 & 14.5 \\
\hline Pentyl-Fc & 2.01 & 19.8 & 19.2 \\
\hline Decyl-Fc & 3.09 & 695 & 31.5 \\
\hline
\end{tabular}

The kinetic constant values obtained in our samples are lower that the values reported in studies with porphyrins and derivates of ferrocene bonded to silicon electrodes ${ }^{21,42}$ as well as those from the monolayers of alkanethiols functionalized with ferrocene molecules bonded to gold electrodes ${ }^{38-40}$. However, there are some studies of immobilized ferrocene molecules on gold electrodes with kinetic constants similar to those reported in our study ${ }^{32,43}$. The above, indicates that in similar conditions, there is a great difference among the kinetic constants reported in electrode-molecule systems.

On the other hand, the results obtained in our three systems, show no evident dependence between the apparent kinetic constant value and the length of the alkyl chain employed for the union between the electrode and the ferrocene molecule. Considering, the crystallographic orientation of the silicon electrode ( $\mathrm{Si}$ (100), producing a dihydride surface), the length of the alkyl chains and the angles between the electrode surface and alkyl chains, the real distance between the electrode surface and the redox molecule can be determined. Thus, the distances obtained are $2.6 \AA, 4.06 \AA$ and $7.74 \AA$ for the chains of 3,5 and 10 carbon atoms, respectively. Which ever the case, these will be the correct distances in highly compact layers, thus avoiding the movement of the chain on the electrode surface. By employing scanning electrochemical microscopy (SECM), with a p-type conducting silicon substrate together with alkyl monolayers, Ghilane et al. ${ }^{44}$ determined that the electron transfer between different redox species in solution and the silicon surface through the organic layer depends on the number of carbon atoms in the chain. In this case, the interfacial electron transfer occurs through electron tunneling across the organic layer (long-rate transfer). However, all the studied cases present a low coverage and therefore the chains are not static on the electrode surface. This results in a "bonded diffusion" regime of the redox centres ${ }^{45}$ avoiding the blocking effect of the compact organic layer. In this situation, the long-range electron transfer is not significant, and the system is controlled by other processes which are different to this. According to our systems and the results obtained, electron hopping is the most feasible process in order to control the electron transfer in these systems. Electron hopping between adjacent ferrocene molecules has been recently verified, using SECM on a non conducting silicon substrate ${ }^{46}$. In this study, the monolayer was densely compact and the electron transfer was very fast thus preventing its detection. This way, the electrochemical process will be influenced by the electrode-molecule distance.

In the studied systems, due to the low coverage, the distance between adjacent redox centers can be big. Regardless of this, the system has an adequate coverage that permits both, the movement of the molecules and the electron transfer between neighbor molecules. Thus, the apparent rate electron transfer is practically the same, being independent of the number of carbon atoms employed in the bondage between the electrode surface and the ferrocene molecule. When a high coverage is reached, the movement of the chains is restricted, and the bonded diffusion regime is practically inexistent. In this last case, the electrochemical process is controlled by the long-range electron transfer and will be influenced by the electrode-molecule distance. Previous studies, where the surface coverage is similar to the levels shown in this study, have reported electron hopping between neighbor atoms of iron and ruthenium in mixed incomplete monolayers ${ }^{47}$. According to the equations developed by Blauch and Savéant ${ }^{45}$ for a system controlled by electron hopping in a bonded diffusion regime (where the movement of the molecules is faster than electron hopping), the fittings, in effect, show negative deviation in a situation of very low coverage. This, because of the inability of the molecules to interact with their nearest neighbors. However, when the molecules are not static on the electrode and present a certain degree of movement (limited physical displacement) the interaction of the molecules will only occur when their effective areas overlap. The above occurs roughly when the following equation takes place:

$$
X>\frac{\delta^{2}}{3 \pi \lambda^{2}}
$$


where

$\mathrm{X}=$ Degree of coverage in a surface with electroactive molecules

$\delta=$ Distance between adjacent redox centers at the moment of electron transfer. $\mathrm{d}$ is similar to the diameter of the employed molecules

$\lambda=$ Range of molecular motion allowed in the case of molecules bonded to the electrode surface.

Assuming that $\delta$ is similar to the diameter of the ferrocene molecules $(6.4$ $\AA)$ and that $\lambda$ is approximate to the length of the employed alkyl chains, the conditions for electron hopping in molecules that are fixed but not immobile on the electrode surface can be determine. Using the coverage obtained through cyclic voltammetry, it is possible to observe that this condition is reached in every studied case. The above mentioned is summarized in Table 3.

Table 3: Relationship between minimum coverage necessary to occurs the electron hopping $\left(\Gamma_{\mathrm{L}}\right)$ determinate from the equation 1 and the coverage reached in each system see in this study.

\begin{tabular}{|ccccc|}
\hline Molecule & $\delta / \AA$ & $\lambda / \AA$ & $\Gamma_{\mathbf{L}} / \mathbf{\%}$ & $\Gamma_{\exp } / \mathbf{\%}$ \\
\hline Propyl-Fc & 6.4 & 4.40 & 22.4 & $15.6-22.6$ \\
\hline Pentyl-Fc & 6.4 & 6.90 & 9.12 & $17.5-25.5$ \\
\hline Decyl-Fc & 6.4 & 13.3 & 2.46 & $37.6-41.7$ \\
\hline
\end{tabular}

Considering the approximations used, it can be observed that all our systems comply with the requirements that allow the electron transfer to be determined by electron hopping between adjacent molecules under a regime of bonded diffusion. Thus, the electron transfer is practically the same, independent of the number of carbon atoms employed in the bonding between the electrode surface and the ferrocene molecule.

\section{CONCLUSIONS}

Throughout the synthesis presented in this study, by changing the alkyl chain length employed in the silicon-ferrocene bond, from 3 to 10 carbon atoms, different hybrid semiconductor-molecules have been obtained. XPS analysis confirms the presence of ferrocene molecules on the electrode surface and $\mathrm{Si}-\mathrm{C}$ bond. Electrochemical analysis has proved that in every studied case, incomplete monolayers have been formed. The electron transfer plainly shows to be independent of the length of the alkyl chain used, because of the low coverage obtained in our systems. Thus, a regime of bonded diffusion becomes feasible, where the electrodic process is controlled through electron hopping between adjacent redox molecules. The systems studied are consistent with the equations developed by Blaunch and Savéant, this way confirming the above mentioned.

\section{REFERENCES}

1. J. M. Buriak, Chem. Rev. 102, 1271, (2002).

2. S. F. Bent, J. Phys. Chem. B 106, 2830, (2002).

3. S. Forrest, MRS Bull. 26, 108, (2001).

4. S. R. Forrest, Chem. Rev. 97, 1793, (1997).

5. A. Ulman, Chem. Rev. 96, 1533, (1996).

6. M. R. Lindford, C. E. D. Chidsey, J. Am. Chem. Soc. 115, 12631, (1993).

7. M. R. Lindford, P. Fenter, P. M. Eisenberger, C. E. D. Chidsey, J. Am. Chem. Soc. 117, 3145, (1995).

8. R. L. Cicero, C. E. D. Chidsey, Langmuir 18, 305, (2002).

9. E. Balaur, T. Djenizian, R. Boukherroub, J. N. Chazalviel, F. Ozama, P. Schmuki, Electrochem. Commun. 6, 153, (2004).

10. A. B. Sieval, R. Linke, G. Heij, G. Meijer, H. Zuilhof, E. J. R. Sudhölter, Langmuir 17, 7554, (2001).

11. N. Saito, S. Youda, K. Hayashi, H. Sugimura, O. Takai, Surf. Sci. 532 $\mathbf{5 3 5}, 970,(2003)$.

12. A. Teyssot, A. Fidélis, S. Fellah, F. Ozanam, J. N. Chazalviel, Electrochim. Acta 47, 2565, (2002).

13. S. Fellah, A. Teyssot, F. Ozanam, J. N. Chazalviel, J. Vigneron, A. Etcheberry, Langmuir 18, 5851, (2002).
14. P. Allongue, C. H. de Villeneuve, J. Pinson, F. Ozanam, J.N. Chazalviel, X. Wallart, Electrochim. Acta 43, 2791, (1998).

15. P. Allongue, C. H. de Villeneuve, J. Pinson, Electrochim. Acta 45, 3241, (2000).

16. P. Allongue, C. H. de Villeneuve, G. Cherouvrier, R. Cortés, M. C. Bernard, J. Electroanal. Chem. 550-551, 161, (2003).

17. R. Zanoni, F. Cataruzza, C. Coluzza, E. A. Dalchiele, F. Decker, G. Di Santo, A. Flamini, L. Funari, A. G. Marrani, Surf. Sci. 575, 260, (2005).

18. E. A. Dalchiele, A. Aurora, G. Bernardini, F. Cattaruzza, A. Flamini, P. Pallavicini, R. Zanoni, F. Decker, J. Electroanal. Chem. 579, 133, (2005).

19. F. Decker, F. Cattaruzza, C. Coluzza, A. Flamini, A. G. Marrani, R. Zanoni, E. A. Dalchiele, J. Phys. Chem. B 110, 7374, (2006).

20. Q. Y.Sun, L. C. P. M. de Smet, B. van Lagen, A. Wright, H. Zuilhof, E. J. R. Sudhölter, Angew. Chem. Int. Ed. 43, 1352, (2004).

21. A. A. Yasseri, D. Syomin, R. S. Loewe, J. S. Lindsey, F. Zaera, D. F. Bocian, J. Am. Chem. Soc. 126, 15603, (2004).

22. Z. Liu, A. A. Yasseri, R. S. Loewe, A. B. Lysenko, V. L. Malinovskii, Q. Zhao, S. Surthi, Q. Li, V. Misra, J. S. Lindsey, D. F. Bocian, J. Org. Chem. 69, 5568 (2004).

23. A. A. Yasseri, D. Syomin, V. L. Malinovskii, R. S. Loewe, J. S. Lindsey, F. Zaera, D. F. Bocian, J. Am. Chem. Soc. 126, 11944, (2004).

24. Q. Li, G. Mathur, M. Homsi, S. Surthi, V. Misra, V. Malinovskii, K. H. Schweikart, L. Yu, J. S. Lindsey, Z. Liu, R. B. Dadke, A. Yasseri, D. F. Bocian, W. G. Kuhr, Appl. Phys. Lett. 81, 1494, (2002).

25. Q. Zhao, Y. Luo, S. Surthi, Q. Li, G. Mathur, S. Gowda, P. R. Larson, M. B. Johnson, V. Misra, Nanotechnology 16, 257, (2005).

26. Q. Li, S. Surthi, G. Mathur, S. Gowda, V. Misra, T. A. Sorenson, R. C. Tenent, W. G. Kuhr, S. Tamaru, J. S. Lindsey, Z. Li, D. Bocian, Appl. Phys. Lett. 83, 198, (2003)

27. B. Fabre, F. Hauquier, J. Phys. Chem. B, 110, 6848, (2006).

28. G. Riveros, G. González, B. Chornik, acepted in J. Brazil. Chem. Soc.

29. S. D. Dietz, W. L. Bell, R. L. Cook, J. Organomet. Chem. 545-546, 67, (1997).

30. S. I. Goldberg, L. H. Keith, T. S. Prokopov, J. Org. Chem. 28, 850, (1963).

31. F. Rebiere, O. Samuel, H. B. Kagan, Tetrahedron Lett. 31, 3121, (1990).

32. S. E. Creager, T. T. Wooster, Anal. Chem. 70, 4257, (1998).

33. S. Dreiner, M. Schürmann, C. Westphal, H. Zacharias, Phys. Rev. Lett. 86, 4068, (2001).

34. F. Karadas, G. Ertas, S. Suzer, J. Phys. Chem. B 108, 1515, (2004).

35. C. Cardinaud, G. Lemperière, M. C. Peignon, P. Y. Jouan, Appl. Surf. Sci. 68, 595, (1993).

36. B. Fischer, M. S. Wrighton, M. Umana, R. W. Murray, J. Am. Chem. Soc. 101, 3442, (1979).

37. F. Zheng, V. Pérez-Dieste, J. L. McChesney, Y. Y. Luk, N. L. Abbott, F. J. Himpse, Surf. Sci. 587, L191, (2005).

38. S. Creager,C. J. Yu, C. Bamdad, S. O'Connor, T. MacLean, E. Lam, Y. Chong, G. T. Olsen, J. Luo, M. Gozin, J. F. Kayyem, J. Am. Chem. Soc. 121, 1059, (1999).

39. J. J. Sumner, K. S. Weber, L. A. Hockett, S. E. Creager, J. Phys. Chem. B 104, 7449, (2000).

40. J. J. Sumner, S. E. Creager, J. Phys. Chem. B 105, 8739, (2001).

41. K. M. Roth, D. T. Gryko, C. Clausen, J. Li, J. S. Lindsey, W. G. Kuhr, D. F. Bocian, J. Phys. Chem. B 106, 8639, (2002).

42. K. M. Roth, A. A. Yasseri, Z. Liu, R. B. Dabke, V. Malinovskii, K.-H Schweikart, L. Yu, H. Tiznado, F. Zaera, J. S. Lindsey, W. G. Kuhr, D. F. Bocian, J. Am. Chem. Soc. 125, 505, (2003).

43. L. A. Hockett, S. E. Creager, Langmuir 11, 2318, (1999).

44. J. Ghilane, F. Hauquier, B. Fabre, P. Hapiot, Anal. Chem. 78, 6019, (2006).

45. D. N. Blauch, J. M. Savéant, J. Am. Chem. Soc. 114, 3323, (1992).

46. F. Hauquier, J. Ghilane, B. Fabre, P. Hapiot, J. Am. Chem. Soc. 130, $2748,(2008)$.

47. C. Hortholary, C. Coudret, C. R. Chimie 11, 702, (2008).

\section{ACKNOWLEDGEMENTS}

This work has been supported by FONDECYT (Chile) project No 1050700 . 